\title{
Maximal CP violation via Higgs-boson exchange
}

\author{
L. Laroura \\ Department of Physics, Carnegic-Mellon University, \\ Pittsburgh, Pennsylvania 15213, U.S.A.
}

DOE/ER/40682--2

DE93 002417

March 6, 1992

\begin{abstract}
The unitarity of the mixing matrix of the charged Higgs $i$ inons, and the orthugonality of the mixing matrix of the neutral Higgs bosons, are used to derive upper bounds on the values of general $\mathrm{CP}$-violating expressions. The bounds are independent of the total number of Higgs fields in any specific model. They allow us to relax the usual assumption of only one Higgs boson being light. It is natural that the CP violation in the exchange of neulral Higgs bosons between bottom quarks be particularly large.
\end{abstract}

There has recently been a renewed interest for models in which CF is violated in interactions mediated by the exchange of Higgs particles. This interest was aroused by Weinberg's $\{1\}$ discovery of a CP-violating three-gluon operator [2] which might give, in such theories, a dangerously large contribution to the electric dipole moment of the neutron. Later, Barr and Zee [3] also discovered a two-loop Feynman graph which in those theories gives a contribution to the electric (or chromo-electric [4]) dipole moment of a fermion, which may be orders of magnitude larger than the one-loop contribution.

All the $C P$-violating quantities in a theory in which $\mathrm{CP}$ is violated via Higgs-boson exchange are linear combinations of some basic CP-odd parameters in the Yukawa couplings. The role of these parameters in the above theories is analogous to the one played by the unique CP-violating parameter $J[5,6]$ in the siandard KobayashiMaskawa $(\mathrm{KM})[7 \mid$ model of $\mathrm{CP}$ violation. Indeed, in both cases $\mathrm{CP}$ violation originates in a unitary mixing of various fields (quarks in the KM model, Higgs bosons in the models studied in this paper), involving complex phases which cannot be absorbed by re-definitions of the fields. However, while in the $\mathrm{KM}$ model we know that $|J|$ cannot be larger than $10^{-1}$, because we have already measured various parametcrs of the relevant mixing matrix (CKM matrix) [7], in the models of CP violation via Higgs-boson exchange the basic CP-odd parameters are totally unknown. In order to estimate the various contributions to the various CP-violating quantities, one of course needs to assume certain values for those parameters. In the absence of any 
experimental information about them, it is natural to use values close to the maximum values that they may take, because those maximum values somehow indicate their order of magnitude. Of course, this theoretical assumption may prove erroneous, because suppressions by small mixing angles may occur. This is indeed what happens in the KM model, where $|J|$ is at least one thousand times smaller than what it might in principle have been $|6|$.

It is thus relevant to find an appropriate definition of "maximal CP violation" in Higgs-boson exchange. Weinberg $\{8\}$ posed this problem, and solved it in the special case of a model in which the electroweak symmetry is broken only by the vacuum expectation values of two Higgs doublets. This is the simplest case in which there may be ('T' nonconservation via. Iliggs-boson exchange. It remains to be seen whether and how the results obtained by Weinberg may be genteralized to a more complicated model. In the absence of any experimental knowledge of the Higgs sector, there is no reason to suppose that that sector is just the minimal one which allows for CP violation.

The issue is complicated by the fact that we do not know how many Higgs bosons might exist. We may compare the situation once more to the one of CP violation in the $\mathrm{KM}$ model. In that case, if there are three generations of fermions there is only one basic CP-odd parameter $J$, and it is natural to define maximal C.P violation as being the situation in which that parameter is maximal [6]. But, if there are more generations of fermions, the number of independent CP-odd parameters in the CKM matrix increases drastically [9], and one does noc know any more how to define maximal CP violation. Similarly, if CP violation is mediated by the exchange of a lot of Higgs bosons, it is not clear whether it is possible to find an effective upper bound to the amount of $\mathrm{CP}$ violation in a given process. The inasses of all those Higgs bosons are unknown, and we do not know whether the effects of the exchange of the various lliggs bosons might interfere constructively, and thus generate a $\mathrm{CP}$ violation much larger than the one obtainable from the exchange of only one boson. Previous authors have avoided facing directly these problems, by assuming that only one Higgs boson effectively mediates CP violation, while all the other Higgs bosons are very heavy and therefore give negligible contributions.

1 show in this paper that this artificial assumption may be eliminated without any lost of generality. The presence of an arbitrary number of Higgs bosons with arbitrary finite masses does not change the upper bounds on the various CP-violating quantities. This is due to the unitarity constraints already emphasized by Weinberg [8]. These constraints say that $C P$ violation via Higgs-boson exchange disappears when all the Iliggs bosons have equal masses, or equivalently that there is a kind of GIM mechanism |10|, operating with scalars instead of with quarks, suppressing CP violation in these models, just as the quark GIM mechanism suppresses CP violation in the KM model. I show that a further consequence of this scalar GIM mechanism is that the maximal CP violation in a given diagram depends only on the masses of two particular Higgs bosons (of the relevant charge sector): the lightest one, and the heaviest one. It does not matter how many Iiggs bosons have intermediate masses, and their exact masses also do not matter. 'Therefore, the assumption used in previous papers, that only one 
Higg boson is light while all others are infinitely heavy. may be substituted by the simpler assumption that there is one Higgs boson which is infinitely heavy. If no such Higgs boson exists, then one knows exactly how the value of maximal CP violation diminishes with the decrease of the mass of the heaviest boson.

I work in the context of the following theory: an electroweak theory with gange group SU(2), ol(1), with an arbitrary number of Higgs multiplets, in arbitrary representations of $S \mathrm{~L}^{\top}(2), \mathrm{OU}(1)$ : the neutral components of these Higgs multiplets have arbitrary vacuum expectation values

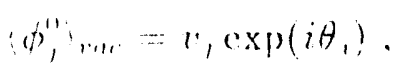

with real and mon-negative $v_{J}$. The restrictive assmmplion is the one of natural flavur conservation: there is only one Higgs doublet, $\phi_{1}$, with Yukawa couplings to the righthanded down-type quarks, and only one lliggs doublet, $\phi_{2}$, with Yukawa conplings to the right-handed up-type quarks. I write these Higgs doublets as

$$
\phi_{1}=\exp \left(i \theta_{i}\right)\left(\begin{array}{c}
\varphi_{i}^{+} \\
v_{j}+\rho_{j}+i \eta_{j}
\end{array}\right) \text {, }
$$

for $j$ equal to 1 or 2 , where $\rho_{j}$ and $\eta_{j}$ are real fields. After having diagonalized the quark mass matrices, the Yukawa interactions are

$$
\begin{aligned}
\mathcal{L}_{1}= & \left(-\frac{\varphi_{1}^{+}}{v_{1}} \overline{u_{1}} V M_{11} d_{l_{i}}+\frac{\varphi_{2}^{+}}{v_{2}} \overline{u_{1 i}} M_{11} V d_{l}+\text { h.c. }\right) \\
& -\frac{\rho_{1}}{v_{1}} \bar{d} M_{1 l} d-\frac{\eta_{1}}{v_{1}} \vec{d} M_{1,} i \gamma_{5} d-\frac{\rho_{2}}{v_{2}} \bar{u} M_{11} u+\frac{\eta_{2}}{v_{2}} \bar{u} M_{11} i \gamma_{5} u .
\end{aligned}
$$

$M_{\text {" }}$ and $M_{\text {t }}$ are the diagonal mass matrices of the up-type and down-type quarks respectively, and $V$ is the CKM matrix. In what concerns $C P$ violation by the exchange of charged Higgs bosons, I will assume that $V$ is real (i.e., $J=0$ ), which follows from the assumptions of natural flavour conservation and spontaneous CP violation [11]. The minimal model of this kind is the Weinberg model of CP violation $[12,13]$.

The Higgs fields in Eq. 3 are not the eigenstates of the Higgs-fields mass matrices. The physical neutral Higgs fields are superpositions of $\rho_{1}, \rho_{2}, \eta_{1}$ and $\eta_{2}$, and of some other real neutral fields which have no Yukawa couplings:

$$
\left(\begin{array}{c}
H_{1}^{\prime \prime} \\
H_{2}^{\prime} \\
I_{1}^{\prime \prime} \\
\cdot \\
\cdot \\
\cdot \\
G^{\prime \prime}
\end{array}\right)=T\left(\begin{array}{c}
\rho_{1} \\
\eta_{1} \\
\rho_{2} \\
\eta_{2} \\
\cdot \\
\cdot \\
\cdot
\end{array}\right) \text {. }
$$

where $T$ is a $m \times m$ orthogonal matrix, $m$ being the total number of neutral Higgs bosons, including the Goldstone boson $G^{\prime \prime} . T$ is the matrix which diagonalizes the mass matrix of the neutral Higgs bosons. As that mass matrix depends on the parameters 
of the Higgs potential, $T$ also depends on that potential, and is therefore in general arbitrary. However, the last row of ' $T$ ' corresponds to the neutral Goldstone boson $G^{\prime \prime}$, which becomes the longitudinal component of the $Z^{\prime \prime}$; that last row is fixed by gauge invariance to be

$$
\left(T_{11, j}\right)=\left(0, v_{1} / v_{11}, 0, v_{2,} / v_{11}, \ldots\right)
$$

$v_{11}=\sqrt{v_{i}}+2 \frac{2}{2}+\ldots$ is a normalization factor, and is related to the mass of the $Z^{\prime \prime}$ by $M^{2}=\left(g^{2}+g^{\prime 2}\right) x_{i 2}^{2} 2$.

Similarly, the physical charged Iliggs particles are superpositions of $\varphi_{1}^{+}, \varphi_{2}^{+}$and of other lliggs fields of charge $+e$ :

$$
\left(\begin{array}{c}
H_{1}^{+} \\
H_{2}^{+} \\
\cdot \\
\cdot \\
G^{+}
\end{array}\right)=11\left(\begin{array}{c}
\varphi_{1}^{+} \\
\varphi_{2}^{+} \\
\cdot \\
\cdot \\
\cdot \\
\cdot
\end{array}\right)
$$

where $W$ is a $n \times n$ unitary matrix, which diagonalizes the hermitian mass matrix of the charged lliggs fields. In general, $W$ is arbitrary and independent of $T$. Its last row corresponds to the charged Higgs boson $\mathrm{G}^{+}$, the longitudinal component of the $\mathrm{W}^{+}$, and is given by

$$
\left(W_{11 j}\right)=\left(\frac{v_{1}}{v_{+}}, \frac{v_{2}}{v_{+}}, \ldots\right) .
$$

The normalization factor $v_{+}=\sqrt{v_{1}^{2}+v_{2}^{2}+\ldots}$ is related to the $W^{+}$mass by $M_{11}^{2}=$ $g^{2} v_{+}^{2} / 2$. If the only Higgs fields with vacuum expectation values (vevs) are in doublets or in singlets of $\mathrm{SU}(2)_{l}$, then $v_{+}=v_{11}$. We know experimentally that $v_{11}$ and $v_{+}$are practically equal, and that their value is approximately $175 \mathrm{GeV}$.

The Yukawa couplings in Eq. 3 may now be written as

$$
\begin{aligned}
\mathcal{L}_{l}= & \frac{1}{v_{+}} \sum_{j=1}^{n-1}\left[H_{j}^{+}\left(\alpha_{j} \overline{u_{l}} V M_{d l} d_{R}+\beta_{j} \overline{u_{R}} M_{u} V d_{l .}\right)+h . c .\right] \\
& +\frac{1}{v_{11}} \sum_{j=1}^{m-1} H_{j}^{\prime \prime}\left(g_{1 j} \bar{d} M_{d d} d+g_{2 j} \bar{d} M_{d d} i \gamma_{5} d+g_{3 j} \bar{u} M_{u} u+g_{1 j} \bar{u} M_{u} i \gamma_{5} u\right) \\
& +\mathcal{L}_{r_{j}},
\end{aligned}
$$

where $\mathcal{L}_{\text {; }}$ contains the couplings of the neutral and the charged Goldstone bosons, which are fixed by gauge invariance, and are immatcrial for our purposes. The coupling constants in Eq. 8 are given by

$$
\begin{gathered}
\alpha_{j}=-\frac{v_{+}}{v_{1}} W_{j 1}^{*}=-\frac{W_{j 1}^{*}}{W_{n 1}^{*}}, \quad \beta_{j}=-\frac{v_{+}}{v_{2}} W_{j 2}^{*}=-\frac{W_{j 2}^{*}}{W_{n 2}^{*}}, \\
g_{1 j}=-\frac{v_{11}}{v_{1}} T_{j 1}, \quad g_{2 j}=-\frac{v_{11}}{v_{1}} T_{j 2}, \quad g_{3 j}=-\frac{v_{11}}{v_{2}} T_{j 3}, \quad g_{1 j}=\frac{v_{11}}{v_{2}} T_{j 11} .
\end{gathered}
$$


adopt a formalism different from the one of Weinberg |8|: I put the C:P violation in the Yukawa couplings, instead of putting it in complex propagators of the Higgs bosons. The Iliggs fields in Eq. 8 are the eigenstates of the mass matrices, and have the usual, real propagators. ('P violation occurs via charged-Higgs exchange if, for any $j, \alpha$, and $\beta$, have different phases. (If they have the same phase, that phase may be absurbed into the definition of $H_{l}^{+}$.) There are therefore $n-1$ CP-violating parameters in charged-lliggs cxchange: $\operatorname{Im}(\alpha, \beta$,$) , with j$ fror 1 to $n$. These parameters are not linearly independent, because the unitarity of $I F$ implies that

$$
\sum_{j=1}^{n-1} \operatorname{lm}\left(\alpha_{,}, \beta_{1}\right) \cdots-\sum_{j=1}^{n} \frac{\operatorname{lm}\left(H_{1} \|_{n 2} H_{j 2} H_{n 1}\right)}{\left|H_{n 1} \|_{n 2}\right|^{2}}=0 \text {. }
$$

For instance, in the Weinberg model, where there exist only two physical charged Iliggs bosons, there is only one basic CP-odd parameter in the charged Higgs sector. The two lliggs bosons have CP-violating effects which interfere destructively, and therefore the CP violation resulting from their simultaneous exchange will vanish if they have equal mass.

Similarly, ('P violation occurs via neutral-Higgs-boson exchange if any one of the real neutral Higgs fields $H_{j}^{\prime \prime}$ has Yukawa couplings with the quarks simultaneously with and without the Dirac matrix $\gamma_{\overline{5}}$, for then no definite CP parity for that neutral Higgs field can be defined. There are therefore $4(m-1)$ CP-violating quantities in the neutral-Higgs-boson sector: $\left(g_{1}, g_{2, j}\right),\left(g_{1, j} g_{1, j}\right),\left(g_{2,} g_{3 j}\right)$ and $\left(g_{3, j} g_{1, j}\right)$, with $j$ from 1 to $m-1$. Because the matrix $T$ is orthogonal, and $T_{m 1}=T_{m ! 3}=0$ (see Eq. 5), these quantities are not linearly independent; they satisfy

$$
\sum_{j=1}^{m-1} g_{1}, g_{2, j}=\sum_{j=1}^{m-1} g_{1 ;} g_{1 j}=\sum_{j=1}^{m-1} g_{2 j} g_{3 j}=\sum_{j=1}^{m-1} g_{3 j} g_{1 j}=0 \text {. }
$$

Therefore, CP violation via the interchange of neutral Higgs bosons vanishes if all those bosons have the same mass.

It should be emphasized that, in specific models, due to a constrained Higgs potential, it may happen that the various CP-violating parameters obey further relations beyond the ones in Eqs. 11 and 12. The Weinberg model is an example of this. In that model, the mass matrix of the three charged Higgs bosons is the most general possible, but the mass matrix of the six real neutral Higgs fields [13] is not as general as possible; in particular, the mass matrix elements connecting $\rho_{1}$ with $\eta_{1}$, and $\rho_{2}$ with $\eta_{2}$, vanish. Also, the mass matrix elements connecting $\rho_{1}$ with $\eta_{2}$ on the one hand, and $\rho_{2}$ with $\eta_{1}$ on the other hand, are symmetrical. As a consequence, in that model there are three further relations among the CP-violating quantitics in the neutral sector beyond the ones in Eqs. 12:

$$
\begin{aligned}
& \sum_{j=1}^{5} M_{j} g_{1, j} g_{2, j}=0, \\
& \sum_{j=1}^{j} M_{j} g_{3 j} g_{1 j}=0,
\end{aligned}
$$




$$
\sum_{j=1}^{5} M_{j}\left(g_{1, j} g_{1 j}-g_{2, j} g_{3 j}\right)=0
$$

where $M_{J}$ is the mass of the $j^{\prime \prime}$ neutral Higgs particle. In this paper I will not take into account the possible existence of conditions among the CP-odd quantities, like the ones in Egs. 13, which are the consequence of a constrained Higgs potential.

At this point, I want to refer to the further constraints on CP violation via neutralHiggs exchange which Weinberg $|8|$ has derived. Weinberg considered the possibility that the only revs which break $\mathrm{SU}(2), \otimes \mathrm{U}(1)$ are $v_{1}$ and $v_{2}$ (all the other scalar fields either have a zero vev or are in singlets of the electroweak gauge group.) In that case, the only non-zero matrix elements in the last row of $T$ are $T_{m: 2} v_{1} v_{n}$ and $T_{m 1}=v_{2} / v_{11}$. The orthogonality condition between the $m$ 'th row of $T$ and any other row of $T$, say the $j$ 'th one, simply reads $v_{1} T_{j 2}+v_{2} T_{j 1}=0$. This leads to further relationships between the "a priori" independent C.P-violating products:

$$
\begin{aligned}
& \left(v_{1}\right)^{2} g_{1 j} g_{2 j}=\left(v_{2}\right)^{2} g_{1 j} g_{1 j}, \\
& \left(v_{1}\right)^{2} g_{2, j} g_{3 j}=\left(v_{2}\right)^{2} g_{3:} g_{1 j},
\end{aligned}
$$

for any $j$ between 1 and $n-1$. These equations were derived (using a different formalism) by Weinberg, and have been used in the papers on the diagram of Barr and Zee for both the electric [3] and the chromo-electric [4] dipole moments, in order to simplify some expressions. I want to emphasize that Eqs. 14 cannot be generalized to the case in which there is at least one more vev breaking the electroweak gauge symmetry. They only apply in the particular case studied by Weinberg.

Let us return to the general case, in which there may be more than two vevs breaking $\mathrm{SU}(2)_{l} \otimes \mathrm{U}(1)$, and consider the general form of the CP-violating part of a certain Feynman graph with the exchange of charged Higgs bosons. (I restrict myself in this paper to the case of only one Higgs internal line in the graph; this case encompasses most practical applications.) For instance, we might be considering the diagram for the generation of a quark electric dipole moment by exchange of charged Higgs particles, or the diagram generating the Weinberg CP-violating three-gluon operator from the exchange of charged Iliggs particles. The general form of the result will be

$$
\sum_{j=1}^{n-1} f\left(m_{j}\right) \operatorname{Im}\left(\alpha_{j}^{*} \beta_{j}\right)=-\frac{v_{+}^{1}}{v_{1}^{2} v_{2}^{2}} \sum_{j=1}^{n-1} f\left(m_{j}\right) \operatorname{Im}\left(W_{j 1} W_{n 2} W_{j 2}^{*} W_{n 1}^{*}\right)
$$

where $f\left(m_{1}\right)$ is some function of the mass of the $j^{\prime \prime \prime}$ charged Iliggs particle, $m_{y}$. The function $f\left(m_{l}\right)$ decreases monotonically with increasing $m_{j}$, vanishing for an infinitely large mass. 'The problem at hand is: supposing that we know all the $m_{j}$, what is the maximum possible value for the modulus of the expression in Eq. 15? In that equation, we are concerned with the first two columns of the unitary matrix $W$. The last matrix elements of each of these two columns $\left(W_{n 1}=v_{1} / v_{+}\right.$and $\left.W_{n 2}=v_{2} / v_{+}\right)$are known. The $f$-function coefficients are also assumed to be known. We want to find out how to choose the remaining matrix elements in those two columns in such a way that the 
linear combination of rephasing-invariant imaginary parts in $\mathrm{Eq} .15$ becomes maximal. It is important to realize that, in any case, that linear combination must have a finite maximum value. This is because the columns of a unitary matrix are normalized, and therefore the matrix elements are not free to increase indefinitely. It might however happen that the maximum value were not an analytical and simple function of the f-function coefficionts; fortunately, this is not the case.

I assert that the maximum possible value of the modulus of the expression in Eq. 15 is

$$
f\left(m_{n}\right)-f\left(m_{+}\right) v_{2} \frac{v_{1}^{2}-v_{2}^{2}}{v_{2}} .
$$

Here, $m_{-}$is the mass of the lightest charged Higgs boson, and $m_{+}$is the mass of the heaviest charged Higgs boson. $f(m)-,f\left(m_{1}\right)$ is therefore the greatest of the differences between any two of the $\int$-function coeflicients in Eq. 15.

The first comment concerning Eq. 16 is that, if the only vevs breaking the elcctroweak symmetry happen to be $v_{1}$ and $v_{2}$ (the case studied by Weinberg), then $v_{+}^{2}=v_{1}^{2}+v_{2}^{2}$ and there is no CP violation via charged-Higgs-boson exchange. This is well known.

I call the attention to the fact that Eq. 16 is invariant under the transformation $f(m) \rightarrow f(m)+x$, with $x$ being an arbitrary real number. This should be so, because Eq. 11 implies that the CP-violating quantity in Eq. 15 is invariant under that transfor nation.

A less trivial point concerns the presence of the denominator $v_{1} v_{2}$ in Eq. 16 . We see that the CP violation may be made arbitrarily large, with $v_{+}$being kept fixed, by just letting $v_{1}$ become arbitrarily small. We readily understand that the origin of this apparent paradox is in the denuminators $v_{1}$ and $v_{2}$ present in the Yukawa couplings as they are written in Eq. 3. Those denominators were introduced in order to have the quark mass matrices, instead of the Yukawa coupling matrices, appear in that Eq. 3. Lelting $v_{1}$ tend to zero while keeping the down-type quark masses fixed corresponds to letting the Yukawa coupling constants of the down-type quarks increase indefinitely, which of course leads to an indefinite increase in the possible amount of $\mathrm{CP}$ violation, but is an unacceptable situation (because the Yukawa interactions become strong). There is thus nothing strange or paradoxical in the presence of the denominator $v_{1} v_{2}$ in Eq. 16.

The proof of Eq. 16 is most transparent if it is done in particular examples, and the reader will have no difficulty in seeing that the proof may be generalized to any other particular case. I give the proof for the following particular example: $n=6$, and the live $f$-function coellicients have the values $10,10,6,4$ and 2 ; this means that there are five charged Higgs bosons, the two lightest ones being degenerate.

For simplicity of visualization, I take two rows, instead of two columns, of a $6 \times 6$ unitary matrix. I suppose that the first matrix elements of each of these two rows are known. Because in our problem we are concerned with rephasing-invariant quantities, we may, without loss of generality, use the rephasing freedom to render the whole first row, and the first matrix element of the second row, real and non-negative. I write the 
two rows as

$$
\left(\begin{array}{cccccc}
a & b & c & d & e & f \\
h & j+i r & k+i s & l+i l & m+i u & n+i v
\end{array}\right),
$$

where $a, b, \ldots, v$ are real numbers. I adopt the convention of denoting the square of each of these numbers by the corresponding capital letter: $A=a^{2}, B=b^{2}, \ldots, V=v^{2}$. The two rows of an unitary matrix in Eq. 17 are normalized,

$$
\begin{gathered}
A+B+C+D+E+F=1, \\
H+J+K+L+M+N+R+S+T+U+F=1,
\end{gathered}
$$

and they are orthogonal to each other:

$$
\begin{aligned}
a h+b j+c k+d l+e m+\int n & =0, \\
b r+c . s+d t+e u+f v & =0 .
\end{aligned}
$$

We know $a$ and $h$, and want to fix the other matrix elements in order to maximize the modulus of

$$
\delta:=10(a b h r+a c h s)+6 a d h t+4 a e h u+2 a f h v .
$$

In our example, $f\left(m_{-}\right)=10$ and $f\left(m_{+}\right)=2$. The first step of the proof consists in using Eq. 21 for writing $\delta$ in such a way that $f\left(m_{--}\right)=-f\left(m_{+}\right)$:

$$
\delta=a h|1(b r+c s)-2 e u-4 f v| .
$$

Elementary algebra now yields

$$
\begin{aligned}
\frac{\delta^{2}}{A H=} & (\downarrow B+4 C+2 E+4 F)(4 R+4 S+2 U+4 V)-p_{1} \\
= & 16(B+C+D+E+F)(R+S+T+U+V)-p_{1}-p_{2}, \\
p_{1}:= & 16\left[(b s-c r)^{2}+(b v+f r)^{2}+(c v+f s)^{2}\right] \\
& +8\left[(b u+e r)^{2}+(c u+e s)^{2}+(e v-f u)^{2}\right] \\
p_{2}:= & 16[D T+D(R+S+U+V)+T(B+C+E+F)] \\
& +12 E U+8[E(R+S+V)+U(B+C+F)] .
\end{aligned}
$$

The important point is that both $p_{1}$ and $p_{2}$ are non-negative. We now start from Eq. 20 and use once more elementary algebra to find that

$$
\begin{aligned}
A I= & (B+C+D+E+F)(J+K+L+M+N)-p_{3}, \\
p_{3}:= & (b k-c j)^{2}+(b l-d j)^{2}+\ldots+(b n-f j)^{2} \\
& +(c l-d k)^{2}+\ldots+(c n-f k)^{2}+\ldots+(e n-f m)^{2} .
\end{aligned}
$$

Once more, the important feature of $p_{3}$ is that it is non-negative. The next step is to use the normalization equation Eq. 19, logether with Eqs. 24 and 27, to find

$$
\frac{\delta^{2}}{A H}=16\left[(B+C+D+E+F)(1-H)-A H-p_{3}\right]-p_{1}-p_{2} .
$$


We finally use the other normalization equation, Eq. 18, to obtain

$$
\frac{\delta^{2}}{A H}=16(1-A-H)-16 p_{3}-p_{1}-p_{2} .
$$

We now recall that $a$ and $h$ are assumed to be given. $\Lambda s p_{1}, p_{2}$ and $p_{3}$ are all nonnegative. Lig. 30 tells us that the maximum possible value of $\delta \%$, as a function of $A$ and of $H$, is $16 . H H(1-d-H)$, and that this maximum value is obtained when $p_{1} \quad p_{2}-p_{3}-0$. Together with the orthonormality conditions of the two rows, $p_{1}=p_{2}=p_{3}=0$ implies that those rows are of the form

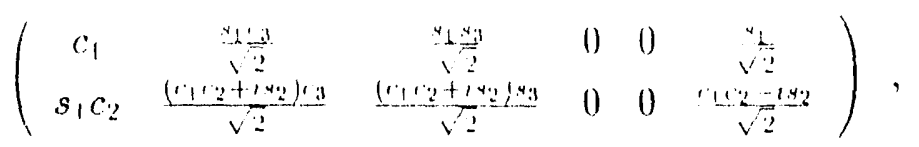

where $c_{1}-\cos \theta_{1}$ and $s_{1}-\sin \theta_{1}$.

Remembering now that we are interested in the siluation in which $a=v_{1} / v_{+}$and $h=v_{2} / v_{+}$, we have that the maximum value of $|\delta|$ obtained is $4 v_{1} v_{2}\left(v_{+}^{2}-v_{1}^{2}-v_{2}^{2}\right)^{1 / 2} / v_{+}^{3}$. The factor 4 is simply, as the clemonstration clearly shows, the difference $\int\left(m_{-}\right)-\int\left(m_{+}\right)$ divided by 2 . Multiplying by the factor $v_{+}^{1} /\left(v_{1}^{2} v_{i}^{2}\right)$ (see Eq. 15), we finally obtain the result stated in Eq. 16. It is clear that the proof, which 1 have performed here for a particular case, can be done in general, whatever the total number of Higgs bosons and whatever the f-function cofficients (i.e., the masses of the bosons). Maximal CP violation is obtained when only the heaviest and lightest Higgs bosons couple to the quarks. Notice the crucial role that the normalization equations of the two rows played in passing from Eq. 27 to Eq.30, and thus in obtaining the upper bound on CP violation.

Let us now analyse the case of CP violation via exchange of neutral Higgs particles. Relevant cases are for instance the diagram for the generation of an electric dipole moment for a down quark (figure 1), or the contribution to the Weinberg three-gluon operator [1] from the exchange of neutral Higgs particles in a loop of top quarks (figure 2 ), or the diagram of Barr and Zee [3] for the chromo-electric [4] dipole moment of a down quark (figure 3 ). In the case of figure 2 , the result has the form

$$
\sum_{j=1}^{m-1} h\left(M_{j}\right) g_{: 3 j} g_{1, j}=-\frac{v_{11}^{2}}{v_{2}^{2}} \sum_{j=1}^{m-1} h\left(M_{j}\right) T_{j 3} T_{j 1},
$$

where $h\left(M_{1}\right)$ is a monotonically decreasing function [14] of the mass of the $j^{\prime \prime}$ neutral lliggs particle, $N_{1}$. The combination $g_{3,3} g_{1}$, in Eq. 32 correponds to CP violation in the exchange of a neutral lliggs boson between two up-type quarks, which CP violation is due to the fact that the Higgs boson couples to the up-type quarks both with and without $\gamma_{, j}$. In the diagram of figure 1 we would have instead a combination $g_{1,}, g_{2,}$, and in the diagram of figure 3 we would have two independent contributions (involving different functions of the mass of the Iiggs boson), one with the combination $g_{1, j} g_{1, j}$ and the other one with the combination $g_{2 j} g_{3, j}$. In any case, the corresponding functions of the masses oh the neutral Higgs particles are always monotonically decreasing. For 
definiteness. I will consider for the moment the case of $C P$ violation via the combinations $g_{3 j} g_{1 j}$, as in $\mathrm{Eq}_{\mathrm{q}}$. 32 . The problem is: if one knows the values of all the $h$-function coefficients, what is the maximum value that one may obtain for the modulus of the expression in Eq. 32? In that equation, we are concerned with the third and fourth columns of the orthogonal matrix $T$. The last matrix elements of each of these two columns $\left(T_{m,}=0\right.$ and $\left.T_{m 1}=v_{2,} v_{n}\right)$ are known.

I assert that the maximum possible value of the modulus of the expression in Eq. 32 is

$$
\frac{h\left(M_{-}\right)-h\left(M_{+}\right) v_{11} \sqrt{v_{11}^{2}-v_{2}^{2}}}{2} .
$$

Lere, $M_{-}$is the mass of the lightest neutral Higgs boson, and $M_{+}$, is the mass of the heaviest neutral Higgs boson. $h\left(M I_{-}\right)-h\left(M_{+}\right)$is the greatest of the differences between any two of the h-function coefficients in Eq. 32 .

I give the proof of this result in a particular case. Take the $2 \times 4$ real matrix

$$
\left(\begin{array}{llll}
a & b & c & d \\
e & f & g & h
\end{array}\right)
$$

and denote as before the square of each number by the corresponding capital letter. Suppose that the two rows of the matrix in $\mathrm{Eq}_{1} .34$ are orthogonal,

$$
a e+b f+c g+d h=0,
$$

and that we want to find out what is the maximum possible value of

$$
\chi:=a e+3 b f+6 c g+13 d h,
$$

subject to the normalization conditions

$$
\begin{aligned}
& A+B+C+D=R, \\
& E+F+G+H=S,
\end{aligned}
$$

where $R$ and $S$ are some two given positive numbers. One solves this problem in the following way. One first uses the orthogonality condition in Eq. 35 to write

$$
\chi=-6 a e-4 b f-c g+6 d h .
$$

Elementary algebra then yields

$$
\begin{aligned}
x^{2}= & 36\left(A+B+C^{\prime}+D\right)(E+F+G+H)-p_{1}, \\
p_{1}:= & 35 C G+30 C(E+H)+30 G(A+D)+32(C F+B G) \\
& +20 B I^{\prime}+12 B(E+I I)+12 F(A+D) \\
& +24(a f-b e)^{2}+6(a g-c e)^{2}+36(a h+d e)^{2} \\
& +4(b g-c f)^{2}+24(b h+d f)^{2}+6(c h+d g)^{2} .
\end{aligned}
$$

The essential point is that $p_{1}$ is non-negative. Using now the normalization Eqs. 37 and 38 , we find that the maximum value possible for $|\chi|$ is $6 \sqrt{R S}$. The factor 6 clearly 
corresponds to the maximum difference of any two coefficients in the original $\chi$ (Eq. 36$)$, divided by $t$ wo. The maximum value of $|\chi|$ is attained when $p_{4}=0$, which means

$$
\begin{aligned}
b=c=f=y & =0, \\
A=l & =\frac{R}{2}, \\
E=H & =\frac{b}{2}, \\
\frac{a}{e}=-\frac{d}{h} & =\sqrt{\frac{R}{S}} .
\end{aligned}
$$

This gives the solution to our problem. Indeed, in the case of Eq. 32 we have two columns of an $m \times m$ orthogonal matrix $T$. As the last matrix element of one of those columns is 0 , we may instead consider that they are orthogonal columns with $m-1$ elements each, with the normalization of one of them being 1 and the normalization of the other one being smaller than 1 . Considering finally the overall factor $v_{11}^{2} / v_{2}^{2}$ in Eq. 32, we reach the result in Eq. 33.

Similarly to Eqs. 32 and 33, we find that the maximum possible values of the three other CP-violating expressions originating in the exchange of neutral Higgs purticles,

$$
\begin{aligned}
& \sum_{j=1}^{m-1} h_{1}\left(M_{j}\right) g_{1 j} g_{2 j}, \\
& \sum_{j=1}^{m-1} h_{2}\left(M_{j}\right) g_{1 j} g_{1 j},
\end{aligned}
$$

and

$$
\sum_{j=1}^{m-1} h_{33}\left(M_{j}\right) g_{2, j} g_{3 j}
$$

are, respectively,

$$
\begin{aligned}
& \frac{h_{1}\left(M_{-}\right)-h_{1}\left(M_{+}\right)}{2} \frac{v_{10} \sqrt{v_{1}^{2}-v_{1}^{2}}}{v_{1}^{2}}, \\
& \frac{h_{2}\left(M_{-}\right)-h_{2}\left(M_{+}\right)}{2} \frac{v_{01} \sqrt{v_{11}^{2}-v_{2}^{2}}}{v_{1} v_{2}},
\end{aligned}
$$

and

$$
\frac{h_{3}\left(M_{-}\right)-h_{3}\left(M_{+}\right)}{2} \frac{v_{11} \sqrt{v_{11}^{2}-v_{1}^{2}}}{v_{1}^{\prime} v_{2}},
$$

respectively.

I now estimate what might be reasonable magnitudes for each of the relevant basic CP-violating parameters. Setting $v_{11}=v_{+}:=v=175 \mathrm{GeV}$, it is reasonable to suppose that, because the mass matrix of the down-type quarks is proportional to $v_{1}$, and the one of the up-type quarks is prorortional to $v_{2}$, then $v_{1} \sim m_{b}$ and $v_{2} \sim m_{1}$. This is the crucial assumption for the results that follow. Because of the presence of denominators 
with $v_{1}$ or $v_{2}$ in the various expressions for maximal CP violation, setting $v_{1}$ to be small of course leads to having some of these expressions become quite large. I emphasize that this is however no dishonest trick, done by someone trying to obtain abnormalously large values for some CP-odd parameters, but rather the usual simple assumption that the Yukawa couplings of the bottom and top quarks should be both of the same order of magnitude. viz. 1.

Setting then $l^{\prime}$ : 5 GeV and $v_{2}=150$ (ieV, we obtain the following numbers. For the ( $P$ violation ria exchange of charged lliggs bosons, the typical order of magnitude of the relerant ('P'-violating parameter will be

$$
J_{+}:=\frac{v v^{2}-v_{i}-v_{i}^{2}}{2 v_{1} v_{2}}=10.5 .
$$

For the $\mathrm{Cl}$ violation via exchange of neutral Higgs bosons, we have, when the neutral lliggs bosons are exchanged between two up-type quarks,

$$
J_{: 31}:=\frac{v \sqrt{v^{2}-v_{:}^{2}}}{2 v_{2}^{2}}=0.35,
$$

and, when they are exchanged between two down-type quarks,

$$
J_{12}:=\frac{v \sqrt{v^{2}-v_{1}^{2}}}{2 v_{1}^{2}}=612.25 .
$$

Finally, if the neutral Higgs bosons are exchanged between an up-type quark and a down-type quark, the basic CP-violating quantity might be, if the $\gamma_{5}$ factor is in the up-quark vertex,

$$
J_{11}:=\frac{v \sqrt{v^{2}-v_{2}^{2}}}{2 v_{1} v_{2}}=10.5,
$$

and, if the $\gamma_{5.5}$ lactor is in the down-quark vertex,

$$
J_{23}:=\frac{v \sqrt{v^{2}-v_{1}^{2}}}{2 v_{1} v_{2}}=20.4 \text {. }
$$

Concerning the value for $J_{+}$in Eq. 52 , it is in order to say that Cheng, in a recent analysis of the Weinberg model of CP violation [15], found precisely that $J_{+}$should be larger than about 9.5 in order for the charged Higgs exchange to be able to account for the obscrved magnitude of 6 . As we sec in Eq. 52, such an order of magnitude for $J_{+}$is perfectly natural, if one takes into account that it is reasonable that the Higgs doublet which gives mass to the down-type quarks should have a vev much smaller than the electroweak scale. This same argument yields for the CP violation via neutral-Higgs exchange the interesting result that we expect $C P$ violation with down-type quarks to be much larger than the one with up-type quarks. This conclusion is against the usual assumptions, done before by various authors, that 1 ) the various $J$ factors in Eqs. 52 to 56 should be all of the same order of magnitude, and 2) CP violation with 
top quarks should be much larger than the one with bottom quarks, because the top couples stronger to the Higgs particles. Indeed, if one compares Eqs. 52, 53 and 54 with the calculations [14] of the various contributions to the Weinberg three-gluon operator, we find that the contribution due to neutral-higgs exchange in a loop of bottom quarks is likely to be much larger than the one with neutral-higgs exchange in a loop of top quarks (in spite of the corresponding $h$-function being much smaller), and that it might be as large as, or larger than, the contribution from charged-higgs exchange (in spite of the contributions from neutral-higgs exchange being suppressed relative to the one from charged-higgs exchange both by smaller $h$-functions and by the $Q(' 1)$ renormalization of the operators). In fact, if the two contributions have opposite signs, they might cancel, and thus the problem claimed by Cheng [15] for the Weinberg model of CP violation, of yielding a very large electric dipole moment for the neutron, would disappear.

Two comments about the limits of applicablity of the upper bounds derived in this paper are in order. The maximum values of $J_{311}, J_{12}, J_{14}$ and $J_{2: 3}$ in Eqs. 53 to 56 cannot all be reached simultaneously. This is so because these four quantities are related with four columns of the orthogonal matrix $T$. In order to reach maximal $C P$ violation in, for instance, $J_{12}$, one has to fix adequately the first two columns of $T$, in particular by setting equal to zero all their matrix elements except those which correspond to the lightest and to the heaviest neutral Higgs particle. But this procedure of course indirectly constrains, due to the orthogonality conditions, the third and fourth columns of $T$, and this in such a way that afterwards the maximum values of $J_{31}, J_{11}$ and $J_{2: 1}$ will not be attainable any more.

The other comment concerns the consequences of further relationships between the matrix elements of $T$, like the ones in Eqs. 13. Such further conditions on. $T$ lead to a smaller value for the maximal $(P$ violation in each of the four neutral channels. lake for instance the upper bound in $\mathrm{Eq}_{\mathrm{q}}$. 33. This upper bound will be realized if the terms $g_{i j} g_{1 j}$ have symmetrical values for the lightest and heaviest neutral Higgs boson, and are zero for all bosons of intermediate mass. But such a situation is incompatible with the second of Eqs. 13. Therefore, the upper bound cannot be reached if that condition applies. There will of course still be an upper bound on CP violation even when that condition applies, but it will be smaller than the one derived in this paper. It turns out that the new upper bound can still be expressed analytically, though in a much more complicated way, as a function of the masses of the various neutral Higgs particles. It does not depend any more just on the masses of the heaviest and lightest neutral Higgs bosons. Depending on the masses, and on the $h$-functions for the particular ('P-violating quantity being analysed, the amount of $C P$ violation that one loses as a conseguence of the conditions in Eqs. 13 may vary a lot. Of course, the most comfortable solution is just to get rid of those extra conditions. In the Weinberg model, they are due to the existence of independent reflection symmetries for the two Higgs doublets $\phi_{1}$ and $\phi_{2}$, which symmetries constrain the Higgs potential. A possible path is to introduce soft terms in that potential which violate those reflection symmetries [16]. This was precisely the path followed by Weinberg in his paper [8]. If such terms 
are not introduced and the conditions of Eqs. 13 apply, both the upper bounds on CP violation in neutral-Higgs exchange that $I$ derived in this paper, and the ones derived by Weinberg [8], will overestimate the pussible amount of CP violation.

I thank Professor G. C. Branco for first calling my attention to the scalar-Gin suppression in CP violation via Higgs-boson exchange. I thank A. K. Gupta for discussions, and Professor L. F. Li for reading the manuscript. This work was supported by the United States Department of Energy, under the contract DE-A(02-76ER-(1)3066.

\section{References}

(1) S. Weinberg, Phys. Rev. Lett. 63 (1989) 2333.

[2] A. Yu. Morozov, Yad. Fiz. 40 (1984) 788 [Sov. J. Nucl. Phys. 40 (1984) 505].

[3] S. M. Barr and A. Zee, Phys. Rev. Lett. 65 (1990) 21.

[4] J. Gunion and D. Wyler, Phys. Lett. B 248 (1990) 170;

D. Chang, W. Y. Keung and T. C. Yuan, Phys. Lett. B 251 (1990) 608.

[5] C. Jarlskog, Phys. Rev. Lett. 55 (1985) 1039.

[6] I. Dunietz, O. W. Greenberg and D. D. Wu, Phys. Rev. Lett. 55 (1985) 2935.

[7] M. Kobayashi and T. Maskawa, Prog. Theor. Phys. 49 (1973) 652.

[8] S. Weinberg, Phys. Rev. D 42 (1990) 860.

[9] F. J. Botella and L. L. Chau, Phys. Lett. B 168 (1.986) 97.

[10] S. L. Glashow, J. Iliopoulos and L. Maiani, Phys. Rev. D 2 (1970) 1285.

[11] G. C. Branco, Phys. Rev. Lett. 44 (1980) 504.

[12] S. Weinberg, Phys. Rev. Lett. 37 (1976) 657;

G. C. Branco, Phys. Rev. D 22 (1980) 2901.

[13] N. G. Deshpande and E. Ma, Phys. Rev. D) 16 (1977) 1583.

[14] D. A. Dicus, Phys. Rev D 41 (1990) 999.

[15] H.-Y. Cheng, Academia Sinica (Taiwan) report IP-ASTL-03-91 (1991), to be published in Int. J. Mod. Phys. A.

[16] G. C. Branco and M. N. Rebelo, Phys. Lett. B 160 (1985) 117. 


\section{FIGURE CAPTIONS}

Figure 1: One-loop diagram giving an electric dipole moment to the down quark from the exchange of neutral Higgs particles $H^{\prime \prime}$. The Yukawa coupling in one of the vertices has a unit matrix in spinor space, and in the other vertex it has a $\gamma_{i}$ matrix.

Figure 2: 'I'wo-loop diagram giving a chromo-electric dipole moment to a gluon (Weinberg operator), from the exchange of neutral Higgs particles in a top-quark loop.

Figure 3: 'Two-loop diagram of Barr and Zee giving a chromo-electric dipole moment to the down quark. The $\gamma_{s}$ matrix may be either in the vertex of the neutral Higgs particles with the down quark, or in their vertex with the top quark. Correspondingly, two different functions of the mass of the Higgs particle, multiplying different basic ('P-odd parameters, appear in the result of this diagram. 


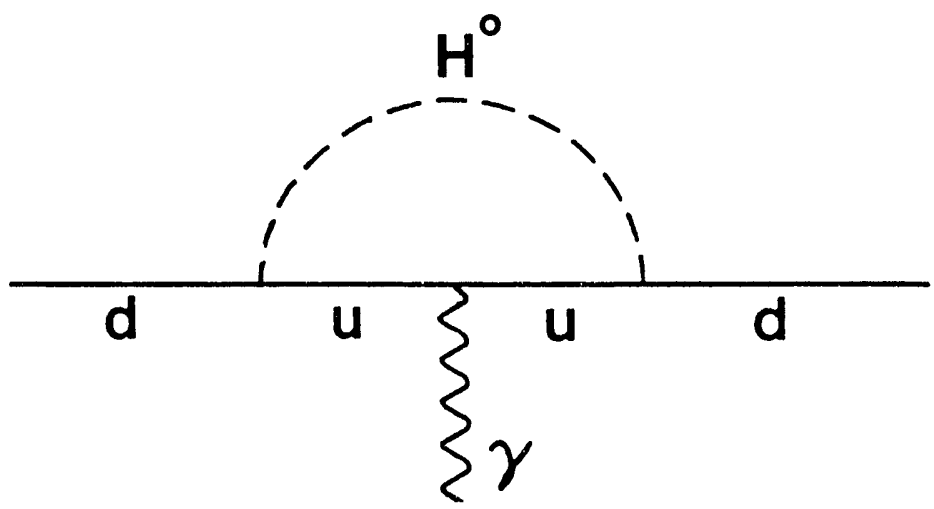

Fig.1

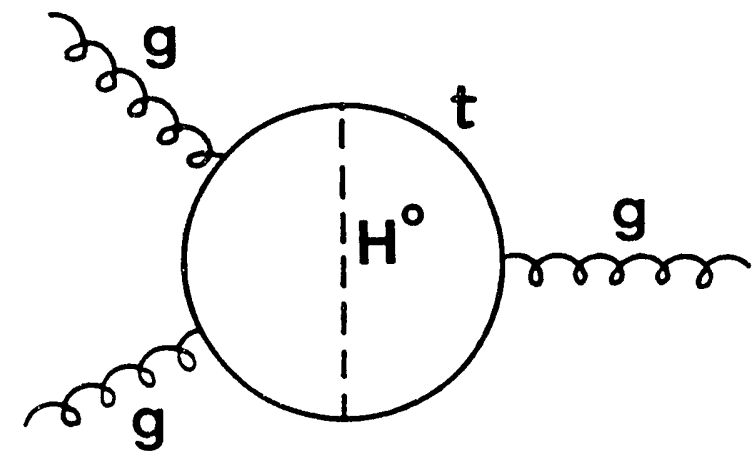

Fig. 2

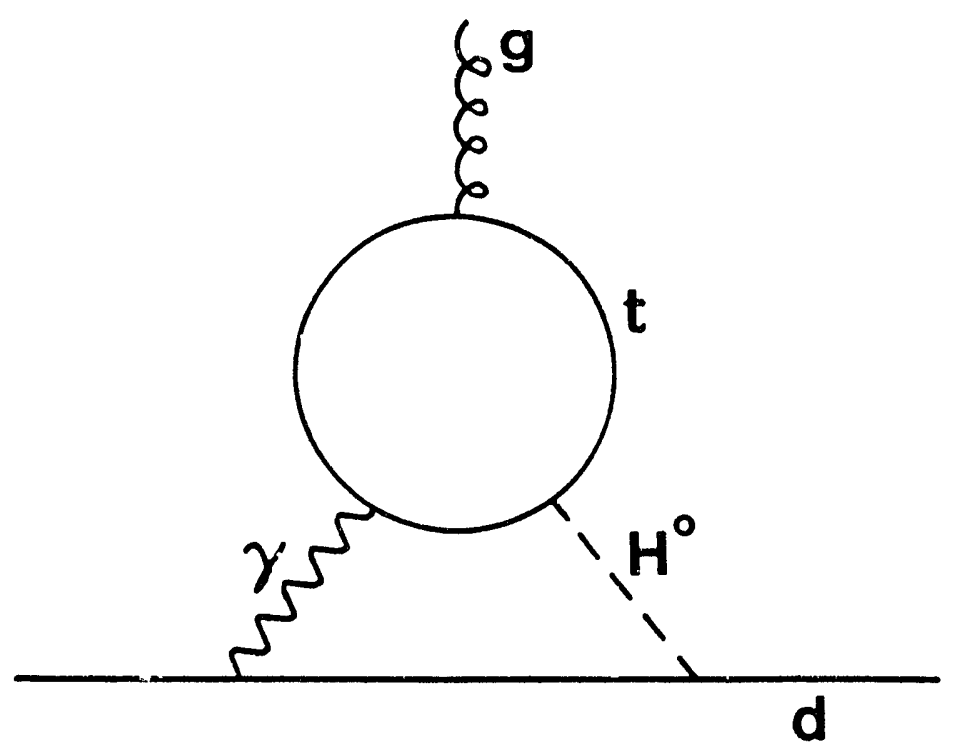

Fig. 3

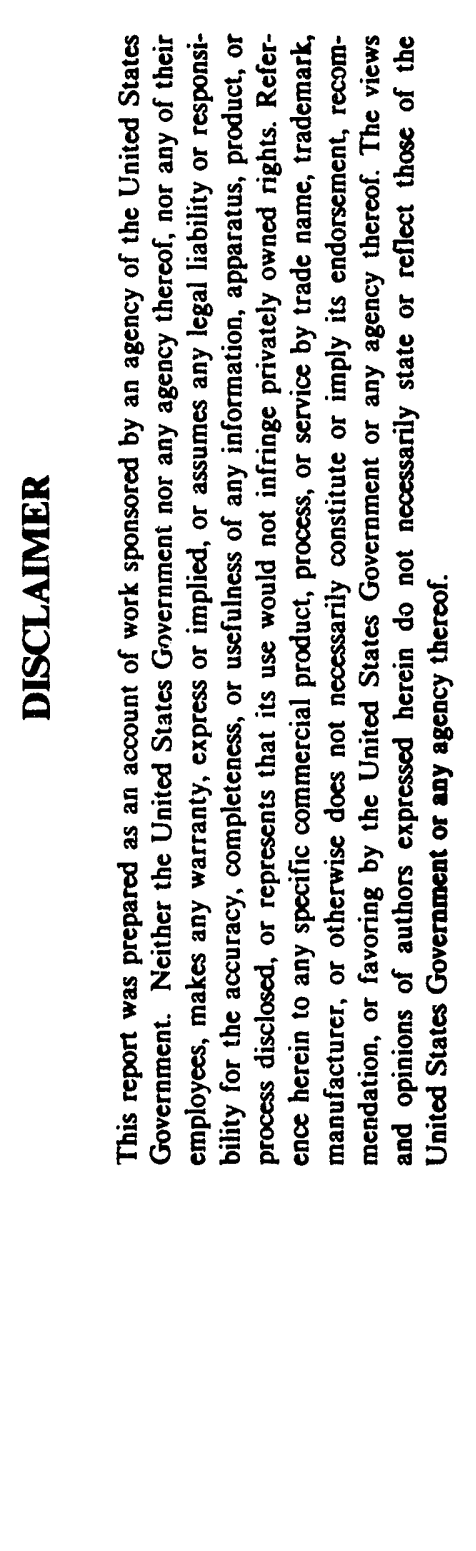



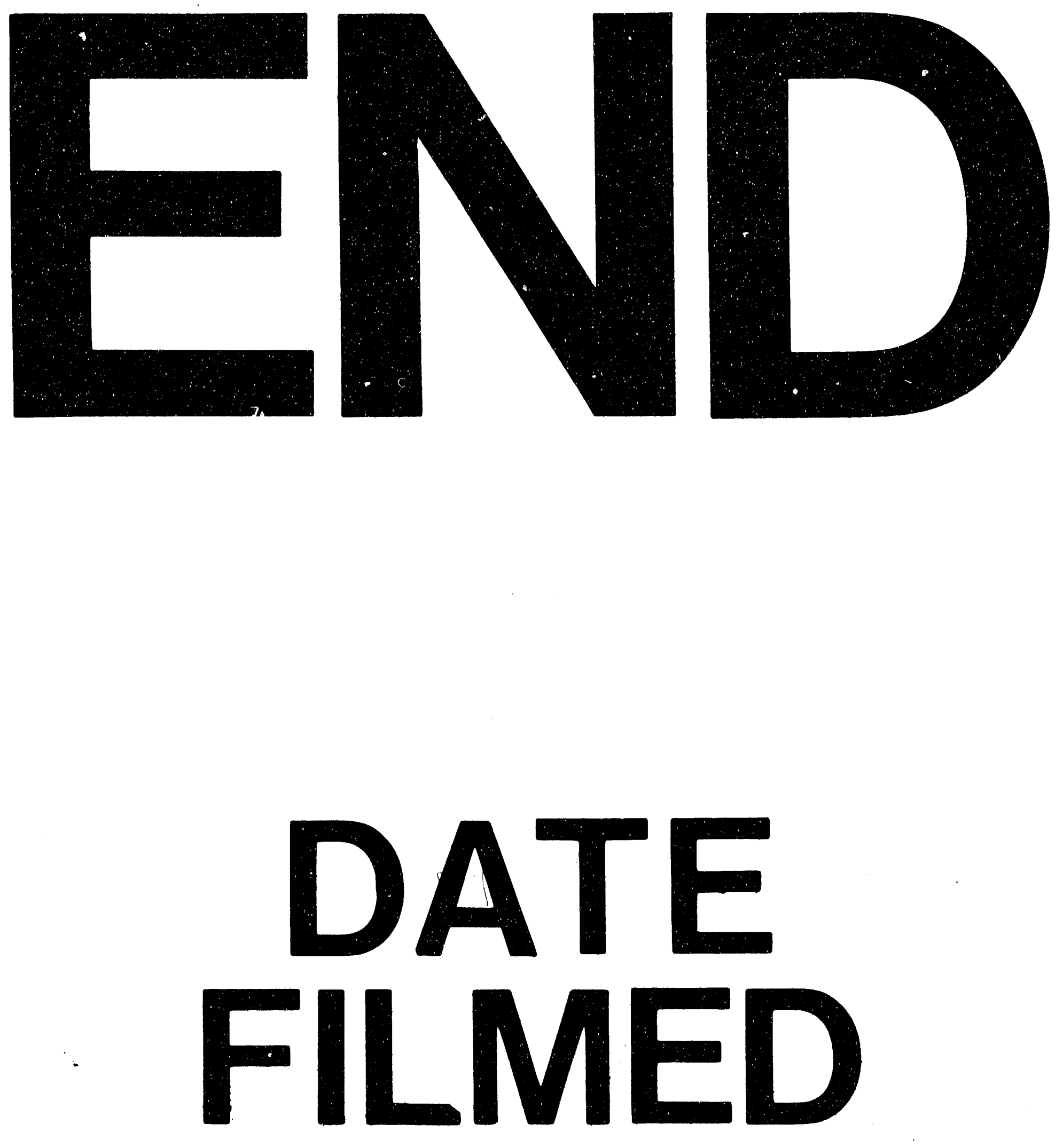

1

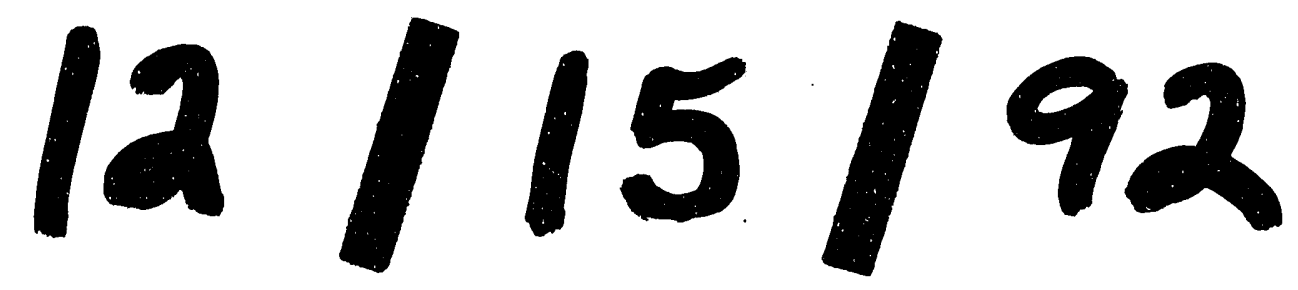


